\title{
Immunoinformatics Based Vaccine Design for Zea M 1 Pollen Allergen
}

\author{
Anamika Basu', Anasua Sarkar², Piyali Basak ${ }^{3}$ \\ 'Department of Biochemistry, Gurudas College, Kolkata, West Bengal, INDIA \\ 2Department of Computer Science and Engg, IEEE Senior member (SMIEEE) Jadavpur University, Kolkata, West Bengal, INDIA. \\ Birector, School of Bioscience and Engineering, Jadavpur University, Kolkata, West Bengal, INDIA.
}

\begin{abstract}
Objective: Zea $\mathrm{m} 1$ is one of the most common aeroallergens, causing allergy. This pollen allergen, present in maize, is responsible for type I hypersensitivity reaction. Despite having available $X$ ray crystal structure of this pollen allergen, no definite vaccine has been developed for allergic disorder in humans. Method: In our present study, an epitope-based peptide vaccine against Zea $\mathrm{m} 1$ pollen allergen, using a combination of $\mathrm{B}$ cell and T cell epitope predictions, followed by molecular docking and molecular dynamics simulation methods are carried out. Here, protein sequences of homologous pollen allergens of Zea $\mathrm{m} 1$ are collected and conserved regions present in them are investigated. Result: From the identified region of the allergenic protein, the peptide sequence KVPPGPNITTNY and the sequence AEWKPMKLSM are considered as the most potential B cell and T cell epitopes respectively. Furthermore, this predicted T cell epitope AEWKPMKLSM interacted with MHC allelic protein HLA$B^{*} 44: 02$ with the lowest $I C_{50}$ value $(7.94 \mathrm{nM})$. This epitope perfectly fitted into the epitope binding groove of alpha helix of $\mathrm{MHC}$ I molecule with lowest energy weighted score -620.0 , showing stability in MHC binding.
\end{abstract}

This epitope also showed a good conservancy of $69.75 \%$ in world population coverage. Conclusion: The epitopes KVPPGPNITTNY and AEWKPMKLSM may be considered as potential peptide for peptide vaccine for pollen allergen after further experimental study.

Key words: Immunoinformatics, Vaccine design, Zea m1 pollen allergen, B cell epitope, T cell epitope, Molecular docking, Molecular dynamics.

Key message: Immunoinformatic study shows that the predicted epitopes KVPPGPNITTNY and AEWKPMKLSM provide in long term and highly specific protective immunity against Zea $\mathrm{m} 1$ pollen allergen during allergic reaction for whole world population

Correspondence

Anamika Basu, Lecturer, Department of Biochemistry, Gurudas College, Kolkata, West Bengal, INDIA.

Phone:9830279500

Email: basuanamikaami@gmail.com

DOI: 10.5530/jyp.2018.10.59

\section{INTRODUCTION}

Allergic diseases are one of the most prevalent health problem along the world. More than $25 \%$ of the world's population is affected by type I hypersensitivity reaction. Among the other causes of allergic reactions, pollen allergens are considered as potential source of hypersensitivity reaction. Aeroallergens, the most common causes of nasobronchial allergy due to contact with grass and tree pollen allergens. ${ }^{1}$ Grass pollen allergens from Zea mays are responsible for the allergic reaction among susceptible individuals. ${ }^{2}$ In the medical science different types of medicines e.g. mast cell stabilizers etc. are used to treat allergic reactions. But several other alternative methods e.g. hypo allergen production, allergen specific immunotherapy, conversion of allergens into vaccines with reduced allergenic activity ${ }^{3}$ etc. are recommended in post genomic era.

Zea $\mathrm{m} 1$ has been crystalized and identified as group I pollen allergen from maize. ${ }^{4}$ By the process of molecular allergen characterization, allergy vaccines can be achieved as recombinant allergens, peptides and genes of allergens. B-cell epitope mapping is a promising method to identifying the main antigenic determinants or epitopes of microorganisms. Epitopebased vaccines have outstanding approach compared to the conventional ones since they are specific in nature, able to avoid unwanted immune reactions, having power to generate long lasting immunity, and are practically cheaper in price. Epitope based peptide vaccine design method is to develop vaccines for various disorders, for example, malaria, ${ }^{5}$ common krait toxin $^{6}$ and zika virus.

\section{MATERIALS AND METHODS}

Retrieval of Zea $m 1$ and identification of conserved regions among homologous pollen allergen of Zea $m 1$

The protein sequence and 3D structure of Zea $\mathrm{m} 1$ allergenic protein were obtained from UniProt knowledgebase. ${ }^{8}$ In SDAP allergen database, ${ }^{9}$ FAO/WHO Allergenicity Rules based on sequence homology for full FASTA alignment for fasta sequence of $2 \mathrm{HCZ}$ showed 50 sequences of allergens with an E score higher than 0.01. Alignment was made with FAST 3.45. ${ }^{10}$ Different protein sequences of pollen allergens were selected and retrieved from Swiss-prot ${ }^{11}$ in FASTA format. To find conserved region, retrieved sequences were aligned using Muscle tool 3.8.31 ${ }^{12}$ where $k$-mer clustering was used. In the next step a tree was constructed by the method known as progressive alignment. The evolutionary divergence analysis for all 50 pollen allergens were completed by forming a phylogenetic tree using Phy ML 3.1/3.0 aLRT software. ${ }^{13}$

\section{Potential T- cell epitope Identification $C D+8 T$ - cell epitope prediction}

Linear T-cell epitopes for MHC-I binding for Zea $\mathrm{m} 1$ allergenic protein are identified by consensus methods using Artificial neural network $(\mathrm{ANN})^{14}$ and Stabilized matrix method (SMM) ${ }^{15}$ from tools for MHC -I binding prediction methods of Immune Epitope Database (IEDB) (www. iedb.org). ${ }^{16} \mathrm{SMM}$ algorithm of MHC-1 binding, transporter of antigenic peptides (TAP) transport efficiency and proteasomal cleavage efficiency

This is an open access article distributed under the terms of the Creative Commons Attribution-NonCommercial-ShareAlike 4.0 License, which allows others to remix, tweak, and build upon the work non-commercially, as long as the author is credited and the new creations are licensed under the identical terms. 
are considered to determine the $\mathrm{IC}_{50}$ values for processing prediction of epitopes by MHC-I molecules. ${ }^{17}$ On the basis of low $\mathrm{IC}_{50}$ values, 5 best epitopes bind with specific MHC-1 molecules are designated for further evaluation.

\section{$C D+4 T$-cell epitope prediction}

CD4+ T-cell receptor responses against concerned allergen are done by using Peptide binding to MHC class II molecules software using MHC II binding prediction tool in IEDB analysis resource, including a consensus approach which combines NN-align, SMM-align ${ }^{18}$ and Combinatorial library methods. For this prediction we chose thirty HLA class II alleles from the reference set. For the predicted T-cell epitopes with low percentile rank are identified and their IC50 values for respective alleles are determined by SMM-align method. ${ }^{18}$

\section{Potential B cell epitope identification}

The $\mathrm{B}$ cell epitope is the part of the antigenic polypeptide chain which interacts with immunoglobulin molecules. Various physico- chemical properties e.g. hydrophilicity, flexibility, accessibility, turns, exposed surface, polarity and antigenic propensity of polypeptides chains have been associated with the location of continuous epitopes of antigenic proteins. ${ }^{19}$ Thus, different tools from IEDB (www.iedb.org), including the classical propensity scale methods such as Kolaskar and Tongaonkar antigenicity scale, ${ }^{20}$ Emini surface accessibility prediction, ${ }^{21}$ Parker hydrophilicity prediction, ${ }^{22}$ Karplus and Schulz flexibilty prediction, ${ }^{23}$ Bepipred linear epitope prediction ${ }^{24}$ and Chou and Fashman beta turn prediction tool ${ }^{25}$ are used to predict $B$ cell epitopes of Zea $m$ 1. From the prediction results most common findings are considered a probable $\mathrm{B}$ cell epitope.

\section{Analysis of population coverage}

Population coverage for identified $\mathrm{T}$ cell epitopes is assessed for world population with the help of IEDB population coverage calculation tool. ${ }^{26}$ This tool computes the fraction of individuals predicted to reply to a given set of epitopes with recognized MHC restrictions.

\section{Docking study of B cell and T cell epitopes}

For docking studies, the T cell epitope AEWKPMKLSM and B cell epitope KVPPGPNITTNY are selected and subjected to PEP-FOLD server ${ }^{27}$ for $3 \mathrm{D}$ structure formation. To identify the molecular interactions with specific HLA protein and immunoglobulin E for respective epitopes, docking studies are performed with ClusPro 2.2 web server. ${ }^{28}$ Cluster scores for lowest binding energy prediction are calculated. Modified PDB ID $1 \mathrm{M} 6 \mathrm{O}$ for HLA-B ${ }^{\star} 44: 02$ is used as allelic protein for docking study of T cell epitope. Similarly, 3D structure (PDB ID 4J4P) for immunoglobulin E molecule is used as receptor molecule for $\mathrm{B}$ cell epitope docking analysis.

\section{Molecular simulation study of predicted T cell epitope with HLA allelic protein}

The entire molecular dynamics simulation study for HLA-epitope protein complex is accomplished with MDWeb i.e. Molecular dynamics on $\mathrm{Web}^{29}$ a web server. A solvent box is added to minimize the energy of the system after equilibrating it using NAMD force field method. Then the solvent is heated to $300 \mathrm{~K}$ and after that protein restraints are lowered. A multiple time step algorithm along with a simulation time step $2.0 \mathrm{fs}$ has been chosen. Molecular dynamics simulations of $5 \mathrm{~ns}$ long is performed at constant temperature and pressure. The MD trajectories are drawn for 0.1 ps for analysis. The trajectories are generated from the simulation study are evaluated for the stability by various parameters viz, B factor (atomic fluctuation), RMSD, radius of gyration.

\section{RESULT}

Homologous pollen allergenic sequences retrieval and identification of conserved regions

From SDAP allergen database, ${ }^{9} 50$ homologous allergens are identified using amino acid sequence of pollen allergen Zea $\mathrm{m} 1$ as shown in Table 1, since sequences with $\mathrm{E}$ values $<0.01$ are almost always homologous in nature. All 50 protein sequences are retrieved from SwissProt ${ }^{11}$ and aligned to identify conserved sequences with varying length using Muscle tool 3.8.31 12 in Table 2 .

A phylogenetic tree illustrating the evolutionary relationship among the 50 homologous allergens is depicted in Figure 1.

\section{CD8+ T-cell epitope identification}

IEDB recommended prediction method for MHC-I binding with CD8+ $\mathrm{T}$ cell epitope suggest that the lower the percentile rank the epitope would be good binder. The predicted percentile rank with consensus method and $\mathrm{IC}_{50}$ values with SMM method ${ }^{15}$ are shown in Table 3.

\section{CD4+ T-cell epitope identification}

Peptide binding cleft in MHC -II molecule is wider than that of MHC- I molecule. So, 15 mer epitopes are predicted by consensus method. $\mathrm{IC}_{50}$ values for predicted epitopes along with their MHC II alleles are displayed in Table 4.

\section{Analysis of Population coverage}

IEDB population coverage too ${ }^{26}$ is used to calculate the population coverage of the predicted epitopes. The result for combined method with both class I and II MHC restriction for the whole world and Indian population with the selected MHC I and MHC II alleles is shown in Table 5.

\section{Prediction of B cell epitopes}

Predicted scores for Emini surface accessibility, ${ }^{21}$ Parker hydrophilicity, ${ }^{22}$ Karplus and Schulz flexibility ${ }^{23}$ and Chou and Fashman beta turn ${ }^{25}$ along with their threshold values are exhibited in Table 6.

Prediction scores of above-mentioned parameters for each residue of peptide ${ }^{28} \mathrm{KVPPGPNITTNYN}{ }^{40}$ reveals that this short stretch of antigenic protein can act as linear B cell epitope. Kolaskar and Tongaonkar antigenicity prediction scores ${ }^{20}$ for each residue of that peptide chain confirms KVPPG as antigenic determinant of Zea $\mathrm{m} 1$ allergenic protein. Residue $\mathrm{N}$ at position 40 is not present in sequence of conserved region.

\section{Docking study of $B$ cell and T cell epitopes}

The $\mathrm{T}$ cell epitope ${ }^{209} \mathrm{AEWKPMKLSW}{ }^{218}$ is selected on the basis of its interactions with large number of alleles and lowest $\mathrm{IC}_{50}$ value with HLA$\mathrm{B}^{\star}$ 44:02 MHC I allele. Similarly, ${ }^{28} \mathrm{KVPPGPNITTNY}{ }^{39}$ is designated as most probable $\mathrm{B}$ cell epitope which is also present in conserved region of pollen allergen Zea m 1. Docking studies are performed with these two epitopes with human HLA-B ${ }^{\star}$ 44:02 MHC I molecule and immunoglobulin protein E respectively.

\section{Molecular simulation study of predicted T cell epitope with HLA allelic protein}

The complex structure of predicted T cell epitope with HLA allelic protein is subjected for molecular dynamics study. The $5 \mathrm{~ns}$ MD simulation of HLA-epitope (HLA-B ${ }^{\star} 44: 02$-AEWKPMKLSW) complex is carried out using NAMD force field, following the energy minimization protocol. The stability of the HLA protein with T cell epitope complex with the help of B factor and RMSD value are calculated and shown in Figure $4 \mathrm{a}$ and $4 \mathrm{c}$. From the figure, it can be concluded that the complex (HLA-B ${ }^{\star} 44: 02$ AEWKPMKLSW) is stabilized after $0.5 \mathrm{~ns}$ simulation, is tended to remain in plateau phase thereafter for the rest of the period. The RMSD value of HLA-epitope complex is observed to grow up from $0.1 \AA$ to $0.8 \AA$ 
Table 1: List of homologous allergens of Zea $\mathbf{m} 1$.

\begin{tabular}{|c|c|c|c|c|}
\hline No & Allergen & Sequence ID in SwissProt/NCBI/PIR & Sequence Length & E score \\
\hline 1 & Zea m 1 & P58738 & 269 & $9.0 \mathrm{e}-96$ \\
\hline 2 & Pas n 1.0101 & ACA23876 & 265 & $2.4 \mathrm{e}-81$ \\
\hline 3 & Ory s 1 & AAF72990 & 269 & $4.0 \mathrm{e}-74$ \\
\hline 4 & Zea m 1 & Q07154 & 191 & $1.6 e-71$ \\
\hline 5 & Ory s 1 & AAF72983 & 267 & $2.2 \mathrm{e}-64$ \\
\hline 6 & Ory s 1 & AAF72991 & 267 & $9.3 e-64$ \\
\hline 7 & Phl p 1 & P43213 & 263 & $4.3 e-60$ \\
\hline 8 & Phl p 1.0101 & CAA81613 & 263 & $5.6 e-60$ \\
\hline 9 & Cyn d 1 & O04701 & 246 & $1.6 e-59$ \\
\hline 10 & Hol 11.0102 & CAA93121 & 248 & $3.5 e-59$ \\
\hline 11 & Holl 11 & P43216 & 265 & $4.0 \mathrm{e}-59$ \\
\hline 12 & Holl 11 & CAA10140 & 263 & $1.2 \mathrm{e}-58$ \\
\hline 13 & Cyn d 1 & AAL14078 & 262 & $3.3 e-58$ \\
\hline 14 & Cyn d 1.0203 & AAL14079 & 262 & $4.9 \mathrm{e}-58$ \\
\hline 15 & Cyn d 1.0202 & AAL14077 & 262 & $4.9 \mathrm{e}-58$ \\
\hline 16 & Poa p a & CAA 10520 & 263 & $6.4 e-58$ \\
\hline 17 & Pha a 1 & Q41260 & 269 & $7.4 \mathrm{e}-58$ \\
\hline 18 & Cyn d 1.0204 & AAF80379 & 244 & $9.4 e-58$ \\
\hline 19 & Cyn d 1.0201 & AAK96255 & 244 & $1.8 \mathrm{e}-57$ \\
\hline 20 & Lol p 1.0103 & CAB63699 & 263 & $1.8 \mathrm{e}-57$ \\
\hline 21 & Ory s 1 & AAF72987 & 275 & $2.1 \mathrm{e}-57$ \\
\hline 22 & Lol p 1.0102 & AAA63278 & 252 & $3.5 e-57$ \\
\hline 23 & Lol p 1.0101 & AAA63279 & 263 & $6.0 e-57$ \\
\hline 24 & Lol p 1 & P14946 & 263 & $7.9 e-57$ \\
\hline 25 & Ory s 1 & AAF72984 & 268 & $3.4 \mathrm{e}-56$ \\
\hline 26 & Ory s 1 & AAF72985 & 286 & $2.8 \mathrm{e}-55$ \\
\hline 27 & Tri a ps93 & AAD10496 & 271 & $1.8 \mathrm{e}-54$ \\
\hline 28 & Ory s 1 & AAB61710 & 261 & $2.0 e-54$ \\
\hline 29 & Ory s 1 & Q40638 & 263 & $3.6 e-53$ \\
\hline 30 & Sor h 1.0101 & ABC58726 & 239 & $6.9 \mathrm{e}-53$ \\
\hline 31 & Ory s 1 & AAF72989 & 271 & $3.4 \mathrm{e}-52$ \\
\hline 32 & Ory s 1 & AAF72988 & 327 & $1.5 \mathrm{e}-42$ \\
\hline 33 & Gly m 2 & AAA50175 & 277 & $2.3 e-39$ \\
\hline 34 & Ory s 1 & AAF72986 & 275 & $2.2 \mathrm{e}-36$ \\
\hline 35 & Arat expansin & CAB37496 & 265 & $5.3 e-20$ \\
\hline 36 & Ory s 1 & AAG13596 & 275 & $6.1 \mathrm{e}-18$ \\
\hline 37 & Ory s 1 & BAA85432 & 284 & $3.0 \mathrm{e}-17$ \\
\hline 38 & Cyn d 2 & CAA10346 & 122 & $3.5 \mathrm{e}-0 \mathrm{~s}$ \\
\hline 39 & Dac g 2 & CAA10345 & 122 & $5.9 \mathrm{e}-09$ \\
\hline 40 & Poa p 2 & CAA 10348 & 122 & $5.9 \mathrm{e}-09$ \\
\hline 41 & Phl p 2 & P43214 & 122 & $5.9 \mathrm{e}-09$ \\
\hline 42 & Lol p 2 & P14947 & 97 & $7.0 \mathrm{e}-08$ \\
\hline 43 & Dac g 3 & P93124 & 96 & $1.2 \mathrm{e}-07$ \\
\hline 44 & Lol p 2 & CAA51775 & 88 & $3.3 e-07$ \\
\hline 45 & Phl p 3.0101 & 2JNZ_A & 108 & $1.5 \mathrm{e}-06$ \\
\hline 46 & Dacg 1.0101 & Q7M1X8 & 34 & $2.0 \mathrm{e}-06$ \\
\hline 47 & Lol p 3 & P14948 & 97 & $3.6 \mathrm{e}-06$ \\
\hline 48 & Cyn d 15 & AAP80171 & 112 & $3.7 e-06$ \\
\hline 49 & Ant o 1.0101 & Q7M1X6 & 32 & $2.2 \mathrm{e}-05$ \\
\hline 50 & Tri a 3 & CAA90746 & 118 & $4.6 \mathrm{e}-05$ \\
\hline
\end{tabular}


Table 2: Conserved sequences of homologous pollen allergens with their positions.

\begin{tabular}{cc}
\hline $\begin{array}{c}\text { Conserved sequences of pollen } \\
\text { allergen }\end{array}$ & Position of the selected sequences \\
\hline KVPPGPNITTNYN & $28-40$ \\
TWYG & $49-52$ \\
DNGGACG & $61-67$ \\
CGNVPIFKDGKGCGSCYE & $82-99$ \\
YHFDLSGKAFGSLAKP & $123-138$ \\
FRRV & $154-157$ \\
FHIEKGCNP & $171-179$ \\
\hline
\end{tabular}

Figure 1: A phylogenetic tree with all fifty pollen allergens showing their phylogenetic relationship.

Potential T-cell epitope identification

Table 3: CD8+ T cell epitopes.

\begin{tabular}{|c|c|c|c|c|c|c|}
\hline No. of epitope & Start position & End position & T cell epitopes & Interacting MHC-I alleles & Percentile rank & $\mathrm{IC}_{50}$ \\
\hline \multirow[t]{3}{*}{1.} & 79 & 88 & MTACGNVPIF & HLA-A*68:02 & 0.2 & 27.77 \\
\hline & & & & HLA-A ${ }^{\star 26: 01}$ & 0.15 & 391.81 \\
\hline & & & & HLA-A ${ }^{\star} 32: 01$ & 0.3 & 604.93 \\
\hline \multirow[t]{2}{*}{2.} & 121 & 130 & NYEPIAPYHE & HLA-A ${ }^{\star} 24: 02$ & 0.15 & 40.41 \\
\hline & & & & HLA-A $A^{\star 23: 01}$ & 0.3 & 10.85 \\
\hline \multirow[t]{2}{*}{3.} & 165 & 174 & KYPAGQKIVF & HLA-A $A^{\star 24: 02}$ & 0.15 & 72.19 \\
\hline & & & & HLA-B ${ }^{\star} 07: 02$ & 0.3 & 114.07 \\
\hline \multirow[t]{2}{*}{4.} & 209 & 218 & AEWKPMKLSW & HLA-B ${ }^{\star} 44: 02$ & 0.15 & 7.94 \\
\hline & & & & HLA-B ${ }^{\star} 44: 03$ & 0.15 & 14.40 \\
\hline 5. & 212 & 221 & KPMKLSWGAI & HLA-B ${ }^{*} 07: 02$ & 0.15 & 6.39 \\
\hline
\end{tabular}

Table 4: CD4+ $t$ cell epitopes.

\begin{tabular}{|c|c|c|c|c|c|c|}
\hline No. of epitope & Start position & End position & T cell epitopes & Interacting MHC-II alleles & Percentile rank & $\mathrm{IC}_{50}$ \\
\hline 1. & 79 & 93 & MTACGNVPIFKDGKG & HLA-DRB1*01:01 & 69.23 & 1759.00 \\
\hline \multirow[t]{6}{*}{2.} & 121 & 135 & NYEPIAPYHEDLSGK & HLA-DRB5 ${ }^{\star} 01: 01$ & 6.33 & 403.00 \\
\hline & & & & HLA-DQA1*01:01 & 6.94 & 749.00 \\
\hline & & & & HLA-DRB3*01:01 & 14.85 & \\
\hline & & & & HLA-DPA1*01:03 & 15.14 & \\
\hline & & & & HLA-DRB1*15:01 & 18.96 & 1029.00 \\
\hline & & & & HLA-DRB1*11:01 & 20.33 & \\
\hline \multirow[t]{2}{*}{3.} & 165 & 174 & KYPAGQKIVFHIEKG & HLA-DRB $1^{*} 11: 01$ & 15.51 & 3299.00 \\
\hline & & & & HLA-DRB1*01:01 & 21.87 & \\
\hline \multirow[t]{3}{*}{4.} & 209 & 223 & AEWKPMKLSWGAIWR & HLA-DRB1*12:01 & 30.1 & 74.88 \\
\hline & & & & HLA-DRB1 ${ }^{*} 03: 01$ & 10.35 & 379.8 \\
\hline & & & & HLA-DRB $1^{*} 08: 02$ & 27.81 & 1234.07 \\
\hline \multirow[t]{5}{*}{5.} & 212 & 226 & KPMKLSWGAIWRMDT & HLA-DRB $1^{*} 11: 01$ & 1.18 & 322.00 \\
\hline & & & & HLA-DRB1*09:01 & 4.27 & 498.00 \\
\hline & & & & HLA-DRB $1^{*} 15: 01$ & 5.12 & 746.00 \\
\hline & & & & HLA-DRB1*08:02 & 6.42 & 749.00 \\
\hline & & & & HLA-DRB3*01:01 & 9.37 & 2170.00 \\
\hline
\end{tabular}


Table 5: Population coverage for predicted T cell epitopes.

\begin{tabular}{|c|c|c|c|c|c|}
\hline \multirow[t]{2}{*}{ Predicted T cell epitopes } & \multirow[t]{2}{*}{ Interacting $\mathrm{MHC}$ I alleles } & \multirow[t]{2}{*}{15 mer peptides } & \multirow{2}{*}{$\begin{array}{l}\text { Interacting MHC II } \\
\text { alleles }\end{array}$} & \multicolumn{2}{|c|}{ Population coverage } \\
\hline & & & & World & India \\
\hline \multirow[t]{6}{*}{ AEWKPMKLSW } & HLA-B ${ }^{\star} 44: 02$ & AEWKPMKLSWGAIWR & HLA-DRB1*12:01 & $69.75 \%$ & $83.44 \%$ \\
\hline & HLA-B ${ }^{\star} 44: 03$ & & HLA-DRB1*03:01 & & \\
\hline & & & HLA-DRB $1^{\star} 08: 02$ & & \\
\hline & & & HLA-DRA1 ${ }^{\star} 01: 03$ & & \\
\hline & & & HLA-DRB1*04:01 & & \\
\hline & & & HLA-DRB $1^{*} 11: 01$ & & \\
\hline \multirow[t]{5}{*}{ KPMKLSWGAI } & HLA-B ${ }^{\star} 07: 02$ & KPMKLSWGAIWRMDT & HLA-DRB1*11:01 & $12.78 \%$ & $2.74 \%$ \\
\hline & & & HLA-DRB $1^{*} 09: 01$ & & \\
\hline & & & HLA-DRB $1^{\star} 15: 01$ & & \\
\hline & & & HLA-DRB $1^{*} 08: 02$ & & \\
\hline & & & HLA-DRB $3{ }^{\star} 01: 01$ & & \\
\hline \multirow[t]{3}{*}{ NYEPIAPYHE } & HLA-A ${ }^{\star 24: 02}$ & NYEPIAPYHEDLSGK & HLA-DPA $1^{\star} 01: 03$ & $5.43 \%$ & $1.5 \%$ \\
\hline & HLA-A $A^{\star 23: 01}$ & & HLA-DRB $1^{\star} 15: 01$ & & \\
\hline & & & HLA-DRB1*11:01 & & \\
\hline \multirow[t]{3}{*}{ KYPAGQKIVF } & HLA-A ${ }^{\star 24: 02}$ & KYPAGQKIVFHIEKG & HLA-DRB1*11:01 & $5.43 \%$ & $1.5 \%$ \\
\hline & HLA-A*23:01 & & & & \\
\hline & HLA-B ${ }^{\star 07: 02}$ & & & & \\
\hline \multirow[t]{3}{*}{ MTACGNVPIF } & HLA-A ${ }^{\star} 68: 02$ & MTACGNVPIFKDGKG & HLA-DRB $1^{*} 01: 01$ & $5.82 \%$ & $3.5 \%$ \\
\hline & HLA-A $A^{\star 26: 01}$ & & HLA-DRB1*08:02 & & \\
\hline & HLA-A*32:01 & & & & \\
\hline
\end{tabular}

Table 6: Surface accessibility, hydrophilicity, flexibility, beta turn and antigenicity prediction score for each residue of B cell epitope.

\begin{tabular}{|c|c|c|c|c|c|}
\hline B cell epitope & $\begin{array}{l}\text { Emini surface accessibility } \\
\text { score for each residue } \\
\text { (threshold }=1.000 \text { ) }\end{array}$ & $\begin{array}{l}\text { Parker hydrophilicity } \\
\text { score for each residue } \\
\text { (threshold=1.521) }\end{array}$ & $\begin{array}{l}\text { Karplus and Schulz } \\
\text { flexibility score for each } \\
\text { residue (threshold=0.993) }\end{array}$ & $\begin{array}{l}\text { Chou and Fashman } \\
\text { beta turn score } \\
\text { for each residue } \\
\text { (threshold }=1.035 \text { ) }\end{array}$ & $\begin{array}{c}\text { Kolaskar and } \\
\text { Tongaonkar } \\
\text { antigenicity score } \\
\text { for each residue } \\
\text { (threshold=1.030) }\end{array}$ \\
\hline K & 1.932 & 2.3 & 1.051 & 1.307 & 1.063 \\
\hline $\mathrm{V}$ & 1.932 & 2.3 & 1.053 & 1.307 & 1.063 \\
\hline $\mathrm{P}$ & 1.932 & 2.3 & 1.063 & 1.307 & 1.063 \\
\hline $\mathrm{P}$ & 2.009 & 3.0 & 1.066 & 1.313 & 1.022 \\
\hline G & 0.704 & 1.043 & 1.067 & 1.236 & 1.054 \\
\hline $\mathrm{P}$ & 1.37 & 2.314 & 1.057 & 1.301 & 0.986 \\
\hline $\mathrm{N}$ & 1.278 & 2.757 & 1.044 & 1.221 & 0.964 \\
\hline I & 1.329 & 3.457 & 1.036 & 1.227 & 0.923 \\
\hline $\mathrm{T}$ & 2.105 & 2.371 & 1.029 & 1.167 & 0.964 \\
\hline $\mathrm{T}$ & 2.189 & 3.071 & 1.025 & 1.173 & 0.923 \\
\hline $\mathrm{N}$ & 1.347 & 2.886 & 1.024 & 1.173 & 0.937 \\
\hline $\mathrm{Y}$ & 3.843 & 4.843 & 1.028 & 1.25 & 0.905 \\
\hline $\mathrm{N}$ & 2.8 & 2.671 & 1.031 & 1.25 & 0.903 \\
\hline
\end{tabular}




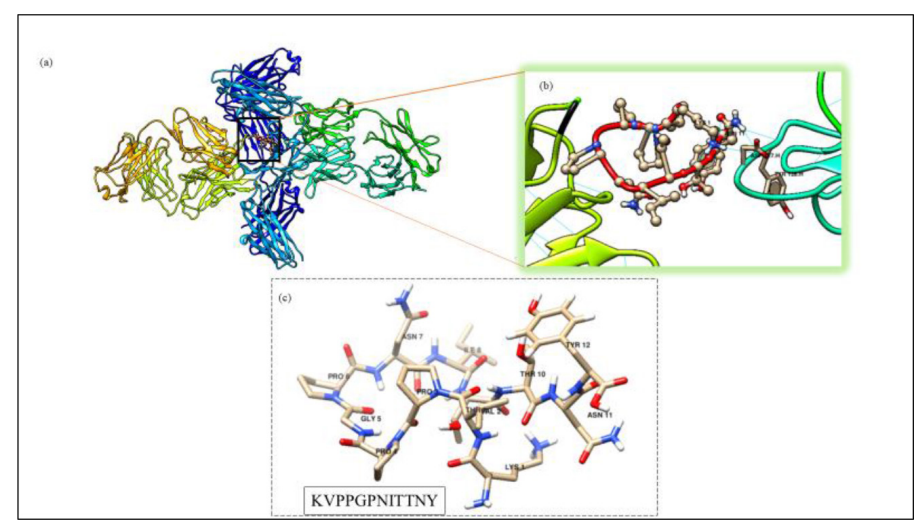

Figure 2: (a) Bound structure of B cell epitope with $\lg E(b) H$ bonding between epitope and CDR region of heavy chain of $\lg E$ (c) $3 D$ structure of $B$ cell epitope. $\mathrm{B}$ cell epitope ${ }^{28} \mathrm{KVPPGPNITTNY}^{39}$ is bound with human immunoglobulin E with lowest energy weighted score -282.5. Hydrogen bonds are formed between side chains of Lys and Asn of epitope and Tyr, Asp of complementarity determining region (CDR) of heavy chain of immunoglobulin molecule (Figure 2 (b)).

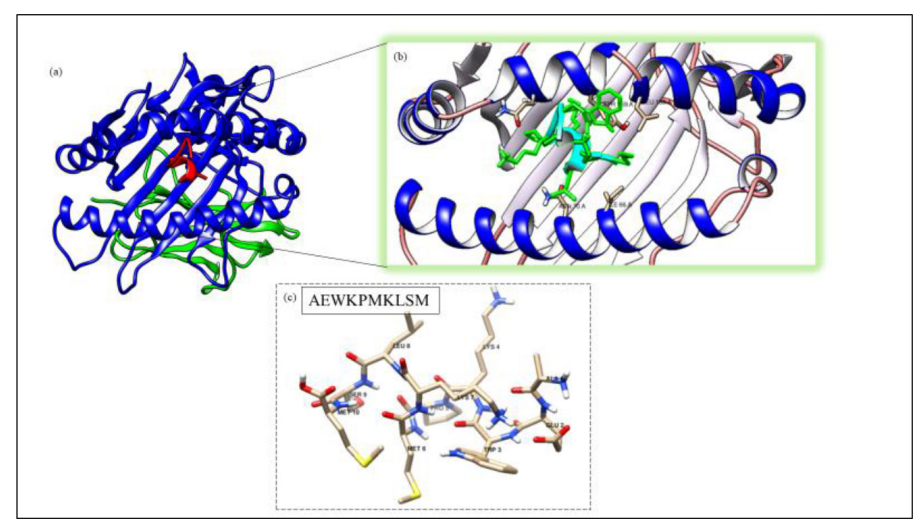

Figure 3:(a) Bound structure of T cell epitope with $\mathrm{MHC} \mathrm{I}$ (b) Interacting residues of epitope and helical groove of alpha chain of $\mathrm{MHC}$ I molecule (c) 3D structure of T cell epitope.

T cell epitope ${ }^{209}$ AEWKPMKLSW ${ }^{218}$ in docking structure with HLA-B*44:02 MHC I molecule, perfectly fitted into the epitope binding groove of alpha helix of MHC I molecule with lowest energy weighted score -620.0 as shown in Figure 3.
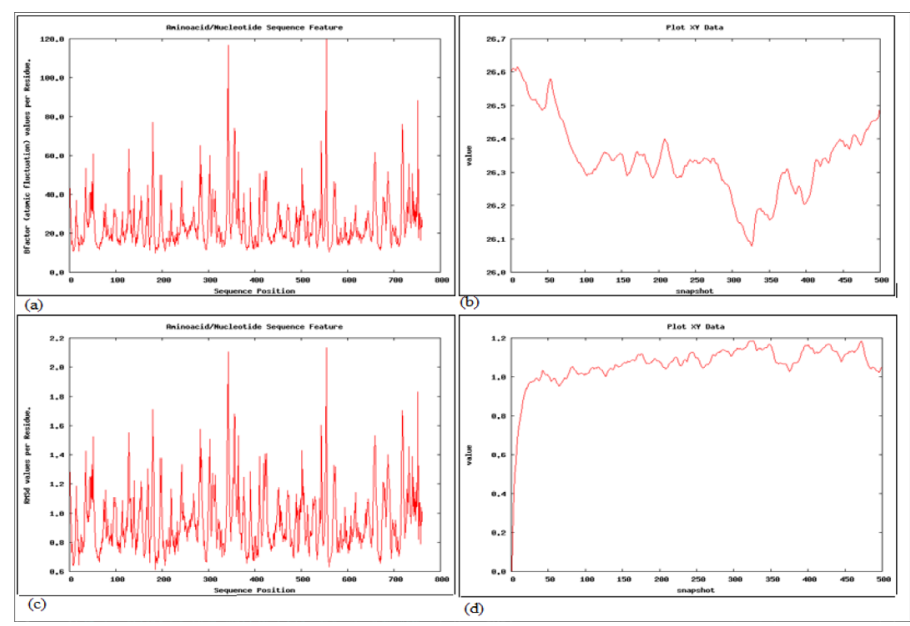

Figure 4: (a) B factor values for individual residues (b) Radius of Gyration for the complex for 5ns (c) RMSD values for individual residues (d) RMSD along trajectory. and remained stable in the range of $1.0 \AA$ to $1.2 \AA$. As seen in Figure $4 \mathrm{a}$, highest $\mathrm{B}$ factor (atomic fluctuation) is observed for SER residue at the position of 342 in HLA-epitope complex, while lowest B factor observed for TRP 237. Similarly, the 342 residue also shows the highest RMSD of $0.915458 \AA$. The plot for radius of gyration of the HLA-epitope complex shows its compactness. The radius of gyration value during $5 \mathrm{~ns}$ simulation slightly varies from 26.1 to 26.6. This means that the HLA-epitope complex is stably folded during simulation study. Hence, all analyses lead to the conclusion that AEWKPMKLSM is the most suitable T cell epitope for peptide-based vaccine design.

\section{DISCUSSION}

In present days, the primary focus of vaccine development mainly depends on peptide-based vaccine design. Here, computational method-based epitope mapping is very efficient method for drug design where the total process is safe, rapid and cost effective. This study incorporates various immunoinformatic, molecular docking and molecular simulation techniques to identify potential epitopes present in Zea $\mathrm{m} 1$ pollen allergen. First of all, fifty homologous allergens from SDAP allergen database ${ }^{9}$ are identified and aligned to identify conserved sequences present in them. Moreover, a phylogenetic analysis shows a closed evolutionary relationship among the homologous allergens. So, it is believed that targeting Zea $\mathrm{m} 1$ pollen allergen for vaccine design against pollinosis, could provide cross reactive reaction data for all homologous allergens.

At the beginning five potent 10 mer $\mathrm{CD} 8^{+} \mathrm{T}$ cell epitopes for $\mathrm{MHC}$-I binding have been predicted from IEDB recommended prediction method. The percentile rank and $\mathrm{IC}_{50}$ values with SMM method, covering all $12 \mathrm{MHC}$ class I supertypes are also predicted. The five most potent epitopes are represented in Table 3 along with their $\mathrm{IC}_{50}$ values.

For MHC I binding prediction, peptides with the lower percentile rank and $\mathrm{IC}_{50}$ values are considered higher affinity for those interacting MHC-I alleles. Therefore, we select AEWKPMKLSW T cell epitope as it binds with HLA-B ${ }^{\star} 44: 02$ with the lowest percentile rank 0.15 and having lowering $\mathrm{IC}_{50}$ value 7.94 for this interacting MHC-I allele. Not only that, AEWKPMKLSW epitope interacting MHC-I allele represents highest population coverage in world as well as in India and henceforth is considered as epitope of choice.

Similarly, due to wider peptide binding cleft in MHC-II molecule than that of MHC-I, 15 mer epitopes are searched by consensus method with their $\mathrm{IC}_{50}$ values and shown in Table 4. For MHC-II binding prediction a 15 mer T cell epitope AEWKPMKLSWGAIWR, starts from 209 position and ends in 223 of allergic protein, shows percentile rank 30.1 with the lowest $\mathrm{IC}_{50}$ value 70.88 during interaction with HLA-BRB1*12:01 MHC-II allele. This result confirms peptide AEWKPMPSW as most suitable T cell epitope for our antigenic protein.

In case of B cell epitope prediction, prediction scores of Emini surface accessibility, ${ }^{21}$ Parker hydrophilicity, ${ }^{22}$ Karplus and Schulz flexibility, ${ }^{23}$ Chou and Fashman beta turn ${ }^{25}$ for each residue of peptide KVPPGPNITTNY starting from 28 position and ending in 39 positions of Zea $\mathrm{m} 1$ protein sequence, reveals that this is the most potential B cell epitope present in this pollen allergen. Furthermore, Kolarskar and Tangaonkar antigenicity prediction scores, confirm our prediction. Lastly, this predicted B cell epitope sequence is also conserved among the fifty homologous allergenic protein sequences.

We also validate our predicted $\mathrm{B}$ cell epitope and $\mathrm{T}$ cell epitope by molecular docking analysis. In Docking study, the B cell epitope KVPPGPNITTNY with protein human immunoglobulin E, forms a stable complex. Similarly, the most suitable $\mathrm{T}$ cell epitope is perfectly fitted with the epitope binding groove of a helix of HLA-B ${ }^{\star}$ 44:02 MHC-I molecule. 
To study the stability of MHC-I protein with peptide AEWKPMKLSW, with the complex is undergone MD simulation study for $5 \mathrm{~ns}$. Plot of radius of gyration shows compactness in MHC-epitope complex. Similarly, the RMSD value during simulation, reaches maxima after $0.5 \mathrm{~ns}$ and remains almost constant during remaining time interval. So, these simulation results evidently demonstrate that the binding complex of $\mathrm{T}$ cell epitope with allelic protein HLA-B ${ }^{\star} 44: 02$, forms a stable complex in thermodynamic environment (Figure 4).

It is one of the important factors in vaccine design that the distribution of HLA allelic protein varies according to the population in different geographic regions of the world. Our selected T cell epitope AEWKPMKLSW is present in $69.75 \%$ of world population and $83.44 \%$ of Indian population. This result indicates that the selected $\mathrm{T}$ cell epitope will specifically bind with the prevalent HLA molecules in the target population in India as well as whole world.

\section{CONCLUSION}

Earlier days, most peptide vaccines have been developed on B cell immunity, however our current study considers both B cell and Tcell epitopes in Zea $\mathrm{m} 1$ pollen allergen. These two epitopes stimulate human immunity during type I hypersensitivity reaction when susceptible individual comes in contact with allergens. These two selected peptides i.e. KVPPGPNITTNY and AEWKPMKLSW show B cell and T cell selectivity, better conservancy among the homologous allergens, highest population coverage of interacting MHC allelic protein. The second epitope depicts significant interaction with MHC-I allele with good affinity. Above all, the predicted epitopes are participated in long term and high protective immunity in peptide vaccine design against Zea $\mathrm{m} 1$ pollen allergen.

\section{ACKNOWLEDGEMENT}

The author acknowledge the software support for prediction and analysis of immune epitopes, provided by Immune Epitope Database Analysis Resource (IEDB).

\section{CONFLICT OF INTEREST}

The authors report no conflicts of interest in this work.

\section{ABBREVIATIONS}

MHC-I: Major histocompatibity complex I; MHC-II: Major histocompatibity complex II; HLA: Human leukocyte antigen complex; MD: Molecular dynamics; NAMD: Nanoscale molecular dynamics; CD8: Cluster of differentiation 8; CD4: Cluster of differentiation 4.

\section{SUMMARY}

Computer based epitope mapping, a state-of-art method is used for vaccine design against Zea $\mathrm{m} 1$ pollen allergen from maize. One of the selected epitope for Zea $\mathrm{m} 1$, is present in the conserved region of other fifty homologous allergenic proteins also. Not only for Zea $\mathrm{m} 1$ pollen allergen, but also for these allergic proteins, the epitope KVPPGPNITTNY is selected as peptide based vaccine for clinical verification against disease pollinosis.

\section{REFERENCES}

1. Pearson WR, Lipman DJ. Improved tools for biological sequence comparison. Proceedings of the National Academy of Sciences. 1988;85(8):2444-8.

2. Schneider M, Bairoch $A$, Wu CH, Apweiler R. Plant protein annotation in the
UniProt Knowledgebase. Plant Physiology. 2005;138(1):59-66.

3. Edgar RC. MUSCLE: Multiple sequence alignment with high accuracy and high throughput. Nucleic Acids Res. 2004;32(5):1792-7.

4. Guindon S, Dufayard JF, Lefort V, Anisimova M, HordijkW, Gascuel O. New algorithms and methods to estimate maximum-likelihood phylogenies: Assessing the performance of PhyML 3.0. Systematic Biology. 2010;59(3):307-21.

5. Balaban J, Bijelic R, Milicevic S. Hypersensitivity to aeroallergens in patients with nasobronchial allergy. Medical Archives. 2014;68(2):86.

6. Cabauatan CR, Ramos JD. Immunoglobulin E-binding reactivities of natural pollen grain extracts from selected grass species in the Philippines. Asia Pacific Allergy. 2012;2(2):136-43.

7. Saroja $\mathrm{CH}$, Lakshmi PK, Bhaskaran S. Recent trends in vaccine delivery systems: A review. Int J Pharma Investig. 2011;1(2):64-74.

8. Yennawar NH, Li LC, Dudzinski DM, Tabuchi A, Cosgrove DJ. Crystal structure and activities of EXPB1 (Zea $\mathrm{m} 1$ 1), a $\beta$-expansin and group-1 pollen allergen from maize. Proceedings of the National Academy of Sciences. 2006;103(40):14664-71.

9. Ullah M, Ghosh T, Ishaque N, Absar N, Hira J. A Bioinformatics Approach for Homology Modeling Binding Site Identification of Triosephosphate Isomerase from Plasmodium falciparum 3D7. J Young Pharmacists. 2012;4(4):261-6.

10. Ashraf KU, Barua P, Saha A, Muhammad N, Ferdoush J, Das D, et al. An immunoinformatics approach towards epitope-based vaccine design through computational tools from Bungarus caeruleus's neurotoxin. J Young Pharmacists. 2014;6(2):35.

11. Hashem MA, Shuvo MA, Arifuzzaman. A Computational Approach to Design Potential Antiviral RNA for 3'UTR Post Transcriptional Gene Silencing of Different Strains of Zika Virus. J Young Pharm. 2017;9(1):23-30.

12. UniProt Consortium. UniProt: The universal protein knowledgebase. Nucleic Acids Research. 2017;45(D1):D158-69.

13. Ivanciuc $\mathrm{O}$, Schein $\mathrm{CH}$, Braun W. SDAP: Database and Computational Tools for Allergenic Proteins. Nucleic Acids Res. 2003;31(1):359-62.

14. Nielsen M, Lundegaard C, Worning P, Lauemoller SL, Lamberth K, Buus S, et al. Reliable prediction of T-cell epitopes using neural networks with novel sequence representations. Protein Sci. 2003;12(5):1007-17.

15. Peters B, Sette A. Generating quantitative models describing the sequence specificity of biological processes with the stabilized matrix method. BMC Bioinformatics. 2005;6(1):132.

16. Vita R, Overton JA, Greenbaum JA, Ponomarenko J, Clark JD, Cantrell JR, et al. The immune epitope database (IEDB) 3.0. Nucleic Acids Res. 2014; 43(D1):D405-12. [Epub ahead of print]

17. Tenzer S, Peters B, Bulik S, Schoor O, Lemmel C, Schatz MM, et al. Modeling the $\mathrm{MHC}$ class I pathway by combining predictions of proteasomal cleavage, TAP transport and MHC class I binding. Cell Mol Life Sci. 2005;62(9):1025-37.

18. Nielsen $M$, Lundegaard $C$, Lund $O$. Prediction of MHC class II binding affinity using SMM-align, a novel stabilization matrix alignment method. BMC Bioinformatics. 2007:8(1):238.

19. Fieser TM, John A, Tainer $H$, et al. Influence of protein flexibility and peptide conformation on reactivity of monoclonal anti-peptide antibodies with a protein a-helix. Proc Natl Acad Sci. 1987;84(23):8568-72.

20. Kolaskar AS, Tongaonkar PC. A semi-empirical method for prediction of antigenic determinants on protein antigens. FEBS Lett. 1990;276(1-2):172-4.

21. Emini EA, Hughes JV, Perlow DS, et al. Induction of hepatitis a virus-neutralizing antibody by a virus-specific synthetic peptide. J Virol. 1985;55(3):836-9

22. Parker JM, Guo D, Hodges RS. New hydrophilicity scale derived from highperformance liquid chromatography peptide retention data: Correlation of predicted surface residues with antigenicity and X-ray-derived accessible sites. Biochemistry. 1986;25(19):5425-32

23. Karplus PA, Schulz GE. Prediction of chain flexibility in proteins. Naturwissenschaften. 1985:72(4):212-3.

24. Pontoppidan JE, Lund $\mathrm{O}$, Nielsen M. Improved method for predicting linear B-cell epitopes. Immunome Res. 2006;2(1):2.

25. Chou PY, Fasman GD. Prediction of the secondary structure of proteins from their amino acid sequence. Adv Enzymol Relat Areas Mol Biol. 1978;47:45-148.

26. Bui HH, Sidney J, Dinh K, Southwood S, Newman MJ, Sette A. Predicting population coverage of T-cell epitope-based diagnostics and vaccines. BMC Bioinformatics. 2006;17(1):153.

27. Shen $Y$, Maupetit J, Derreumaux P, Tufféry P. Improved PEP-FOLD approach for peptide and mini protein structure prediction. J Chem Theor Comput. 2014; 10(10):4745-58.

28. Kozakov D, Hall DR, Xia B, Porter KA, Padhorny D, Yueh C, et al. The ClusPro web server for protein-protein docking. Nature Protocols. 2017;12(2):255-78.

29. Hospital A, Andrio P, Fenollosa C, Cicin-Sain D, Orozco M, Gelpí JL. MD Web and MDMoby: An integrated web-based platform for molecular dynamics simulations. Bioinformatics. 2012;28(9):1278-9. 